\title{
An application of the $\Phi$-functions series method to the integration of seismic modelling
}

\author{
A. Reyes ${ }^{1}$, J. A. Reyes ${ }^{2}$, M. Cortés-Molina ${ }^{2}$, Y. Villacampa ${ }^{2}$ \\ \& F. García-Alonso ${ }^{2}$ \\ ${ }^{1}$ Research Group Mathematical Modeling of Systems, \\ University of Alicante, Spain \\ ${ }^{2}$ Department of Applied Mathematics, Escuela Politécnica Superior, \\ University of Alicante, Spain
}

\begin{abstract}
The interest to improve the response of structures in front of an earthquake has increased in recent years, leading to the investigation of different calculation methods, especially those based on static non-linear analysis to increase accuracy. The non-linear calculation can be approached by means of discrete or continuous models. The discrete models represent the structure by a finite number of degrees of freedom; in this case the movement equations are ordinary differential equations which are resolved by numerical methods.

This paper applies a new method for the numerical integration of SDOF and 2DOF, which is developed from the Scheifele methods. The algorithm integrates the unperturbed problem without truncation error, which represents an advantage in front of the Taylor series. The new method calculates the exact solution of the perturbed problem through a series of functions, whose coefficients are obtained by simple algebraic recurrences involving the perturbation function.

To illustrate the application of the algorithm the resolution of two linear systems is shown; the first one with a single degree of freedom and the second with two degrees of freedom.

Keywords: seismic response, SDOF, 2DOF, numerical solutions, perturbed linear systems of ODEs.
\end{abstract}




\section{Introduction}

In recent years, interest in improving the response of structures to seismic activity has increased dramatically which has in turn led to research into the different methods of calculation. Historically speaking, structural calculations, both in the field of building and civil engineering, have been carried out from a static viewpoint, with a particular focus on those based on non-linear static analysis to increase precision.

The need for non-linear calculation is due to the fact that elastic calculation allows us to obtain the elastic capacity of the structure but not the failure mechanism of the same and, therefore, the redistribution of stresses on the sections.

Non-linear calculation also enables greater detail to be achieved as regards the structural model and, particularly, in formulating a more precise equation to model the movement caused by seismic activity.

Non-linear calculation can be approached from the point of view of continuous or discrete models. Continuous models have distributed parameters, where the movement equations are differential equations in partial derivatives, models whose exact integration is only possible in the case of structures with simple geometry.

Discrete models represent the structure through a finite number of degrees of freedom. In this case, the movement equations are ordinary differential equations that are resolved by numerical methods.

Typical civil engineering structures are always schematised as MDOF. For example, buildings with several floors are analysed using these systems.

For $n$ degrees of freedom, the equation is expressed in matricial fashion:

$$
\begin{gathered}
M \ddot{\boldsymbol{x}}(t)+C \dot{\boldsymbol{x}}(t)+K \boldsymbol{x}(t)=-M a(t) \\
\boldsymbol{x}(0)=\boldsymbol{x}_{\mathbf{0}}=\mathbf{0}, \dot{\boldsymbol{x}}(0)=\dot{\boldsymbol{x}}_{\mathbf{0}}=\mathbf{0}
\end{gathered}
$$

where $M, C$ and $K$ are the mass, damping and stiffness matrices, respectively. The vector column $\boldsymbol{a}(t)$ contains the acceleration values.

These problems are currently resolved by using different software programs that implement numerical methods to calculate the structural response to such oscillatory movements as seismic activity.

This study applies a new method for the numerical integration of these kinds of oscillators and systems, and is based on the methods used by Scheifele [1-5], involving an extension of the Taylor series methods. Said method allows us to express the solution of the system as a series of $\Phi$-functions, which are real functions with values in the ring of the matrices $\mathcal{M}(m, \mathbb{R})[6]$, obtaining the coefficients of the series by recurrences which involve the perturbation function.

The $\Phi$-function series method is able to integrate the perturbed problem exactly, which is an advantage over algorithms based on the Taylor series.

In order to achieve numerical integration of the IVP (1), a linear differential operator is defined, $D+B$, with $B$ being a suitable matrix that allows us to annihilate the perturbation terms, transforming the system into a homogeneous 
second-order system, and managing to integrate it exactly with only the two first $\Phi$-functions of the series method.

To illustrate how the new algorithm is applied, we show the resolution of the single degree of freedom and two degrees of freedom linear system, which model earthquakes.

The precision and efficiency of the $\Phi$-function series method is contrasted with the results obtained by other well-known integrators, such as LSODE, Rosenbrock, Gear, Newmark $\beta$-method and the Wilson $\theta$-method.

\section{Application of the $\Phi$-functions series method to an earthquake modelled by an SDOFa}

The equation of motion (or dynamic equilibrium equation) of a Single Degree Of Freedom (SDOF) is $m \ddot{x}(t)+c \dot{x}(t)+k x(t)=F_{e}(t)$ where $m$ is the floor's mass, $c$ and $k$ are the damping and stiff coefficients, respectively. $F_{e}(t)$ is the external force [7].

The importance of a SDOF resolution lies in that is that best shows the interdependence between structure and its properties and the duration of an earthquake.

Whereas the given structure (Fig. 1), which is not subject to any external force but a movement of the ground due to an earthquake, the elastic force of the columns is expressed through $F_{s}=-k\left(y(t)-u_{g}(t)\right)$, where $y(t)$ and $u_{g}(t)$ are the absolute displacement of the mass and of the ground, respectively.

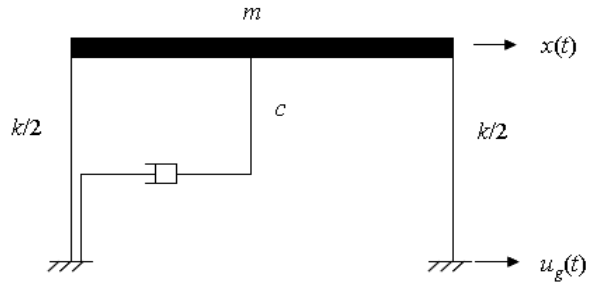

Figure 1: $\quad$ Single Degree of Freedom System (SDOF).

The expression $x(t)=y(t)-u_{g}(t)$ is the relative displacement between the mass and the ground, therefore $F_{s}=-k x(t)$.

Analogously, the damping force is $F_{d}=-c\left(\dot{y}(t)-\dot{u}_{g}(t)\right)=-c \dot{x}(t)$ and the external force is zero.

Applying Newton's second law, $\quad \sum F=m \ddot{y}(t), \quad$ is obtained $m \ddot{x}(t)+c \dot{x}(t)+k x(t)=-m \ddot{u}_{g}(t)$, in standardized form:

$$
\ddot{x}(t)+2 \zeta \omega_{n} \dot{x}(t)+\omega_{n}^{2} x(t)=-\ddot{u}_{g}(t)
$$


where $\omega_{n}=\sqrt{k / m}$ is the undamped natural frequency of vibration and $\zeta=c /\left(2 m \omega_{n}\right)$ is the critical damping ratio.

If $m \ddot{u}_{g}(t)$ is a harmonic forcing function, i.e. $m \ddot{u}_{g}(t)=F_{0} \sin \left(\omega_{0} t\right)$ equation (2) can be expressed:

$$
\ddot{x}(t)+2 \zeta \omega_{n} \dot{x}(t)+\omega_{n}^{2} x(t)=-\frac{F_{0}}{m} \sin \left(\omega_{0} t\right)
$$

at the moment the earthquake occurs, it is very reasonable to assume that the structure is at rest, i.e. $x(0)=0, \dot{x}(0)=0$ and $t \in[0, T]$.

In order to apply the $\Phi$-function series method, the change of variable is affected:

where

$$
x=u_{1}, \dot{x}=u_{2}
$$

$$
\begin{gathered}
\dot{u}_{1}=\dot{x}=u_{2} \\
\dot{u}_{2}=\ddot{x}=-2 \zeta \omega_{n} \dot{x}-\omega_{n}^{2} x-\frac{F_{0}}{m} \sin \left(\omega_{0} t\right)=-2 \zeta \omega_{n} u_{2}-\omega_{n}^{2} u_{1}-\frac{F_{0}}{m} \sin \left(\omega_{0} t\right)
\end{gathered}
$$

The IVP (3) can be expressed as:

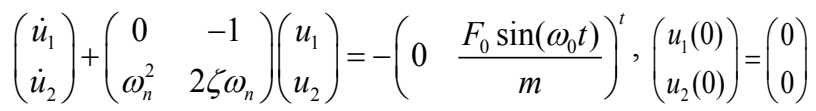

The variable is introduced in order to make easier the elimination the disturbance's function of the IVP (5), following the Steffensen's techniques [8, 9].

$$
\begin{aligned}
u_{3}=- & \frac{F_{0}}{m} \sin \left(\omega_{0} t\right) \text {, obtaining a new IVP. } \\
& \left(\begin{array}{l}
\dot{u}_{1}(t) \\
\dot{u}_{2}(t) \\
\dot{u}_{3}(t)
\end{array}\right)+\left(\begin{array}{ccc}
0 & -1 & 0 \\
\omega_{n}^{2} & 2 \zeta \omega_{n} & 0 \\
0 & 0 & 0
\end{array}\right)\left(\begin{array}{l}
u_{1}(t) \\
u_{2}(t) \\
u_{3}(t)
\end{array}\right)=-\left(\begin{array}{lll}
0 & \frac{F_{0}}{m} \sin \left(\omega_{0} t\right) & \left.\frac{F_{0}}{m} \omega_{0} \cos \left(\omega_{0} t\right)\right)^{t},
\end{array}\right.
\end{aligned}
$$

with $\boldsymbol{u}(0)=\left(\begin{array}{lll}0 & 0 & 0\end{array}\right)^{t}$

To invalidate the function of disturbance, the differential operator $(D+B)$ is applied to (6), where $B$ is the following matrix:

$$
B=\left(\begin{array}{ccc}
0 & 0 & 0 \\
0 & 0 & -1 \\
0 & \omega_{0}^{2} & 0
\end{array}\right)
$$

obtaining the extended IVP,

$$
\begin{gathered}
\left(\begin{array}{l}
\ddot{u}_{1}(t) \\
\ddot{u}_{2}(t) \\
\ddot{u}_{3}(t)
\end{array}\right)+\left(\begin{array}{ccc}
0 & -1 & 0 \\
\omega_{n}^{2} & 2 \zeta \omega_{n} & -1 \\
0 & \omega_{0}^{2} & 0
\end{array}\right)\left(\begin{array}{l}
\dot{u}_{1}(t) \\
\dot{u}_{2}(t) \\
\dot{u}_{3}(t)
\end{array}\right)+\left(\begin{array}{ccc}
0 & 0 & 0 \\
0 & 0 & 0 \\
\omega_{n}^{2} \omega_{0}^{2} & 2 \zeta \omega_{n} \omega_{0}^{2} & 0
\end{array}\right)\left(\begin{array}{l}
u_{1}(t) \\
u_{2}(t) \\
u_{3}(t)
\end{array}\right)=\left(\begin{array}{l}
0 \\
0 \\
0
\end{array}\right) \\
\boldsymbol{u}(0)=\left(\begin{array}{lll}
0 & 0 & 0
\end{array}\right)^{t}, \dot{\boldsymbol{u}}(0)=\left(\begin{array}{lll}
0 & 0 & -\frac{F_{0}}{m} \omega_{0}
\end{array}\right)^{t}
\end{gathered}
$$


which is integrated exactly using the $\Phi$-functions series algorithm described in [6].

\subsection{Resolution of a seismic model (SDOF) by $\Phi$-functions series method}

Choosing the following specific values for the structural variables [7]:

$$
m=1.0 \frac{\mathrm{k} \cdot \mathrm{s}^{2}}{\mathrm{in} .}, \zeta=5 \%, \omega_{n}=2 \pi \frac{\mathrm{rad}}{\mathrm{s}}, F_{0}=10 \mathrm{kip}, \omega_{0}=4 \frac{\mathrm{rad}}{\mathrm{s}} \text { and } T=1 \mathrm{~s} .
$$

The IVP is:

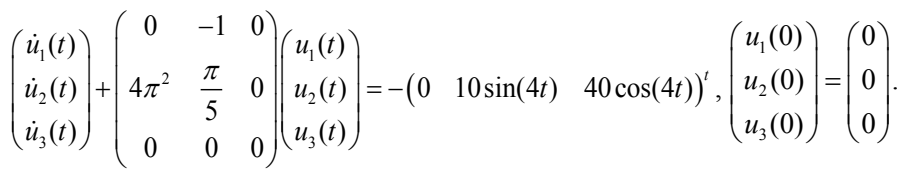

By applying the differential operator, $(D+B)$, is obtained the expanded IVP:

$$
\begin{gathered}
\left(\begin{array}{l}
\ddot{u}_{1}(t) \\
\ddot{u}_{2}(t) \\
\ddot{u}_{3}(t)
\end{array}\right)+\left(\begin{array}{ccc}
0 & -1 & 0 \\
4 \pi^{2} & \frac{\pi}{5} & -1 \\
0 & 16 & 0
\end{array}\right)\left(\begin{array}{l}
\dot{u}_{1}(t) \\
\dot{u}_{2}(t) \\
\dot{u}_{3}(t)
\end{array}\right)+\left(\begin{array}{ccc}
0 & 0 & 0 \\
0 & 0 & 0 \\
64 \pi^{2} & \frac{16 \pi}{5} & 0
\end{array}\right)\left(\begin{array}{l}
u_{1}(t) \\
u_{2}(t) \\
u_{3}(t)
\end{array}\right)=\left(\begin{array}{l}
0 \\
0 \\
0
\end{array}\right), \\
\boldsymbol{u}(0)=\left(\begin{array}{lll}
0 & 0 & 0
\end{array}\right)^{t}, \dot{\boldsymbol{u}}(0)=\left(\begin{array}{lll}
0 & 0 & -40
\end{array}\right)^{t},
\end{gathered}
$$

which is integrated exactly by the following algorithm, particularized for this problem.

$$
\begin{gathered}
\boldsymbol{a}_{0}=\boldsymbol{u}_{0}=\left(\begin{array}{lll}
0 & 0 & 0
\end{array}\right)^{t} \\
\boldsymbol{a}_{1}=\left(\begin{array}{ccc}
0 & 1 & 0 \\
-4 \pi^{2} & \frac{-\pi}{5} & 0 \\
0 & 0 & 0
\end{array}\right) \boldsymbol{a}_{0}-\left(\begin{array}{c}
0 \\
0 \\
40
\end{array}\right)
\end{gathered}
$$

from $\mathrm{k}=1$ up to $\mathrm{n}$ calculates:

$$
\begin{gathered}
\boldsymbol{u}_{k}=\Phi_{0}(h) \boldsymbol{a}_{0}+\Phi_{1}(h) \boldsymbol{a}_{1} \\
\boldsymbol{a}_{0}=\boldsymbol{u}_{k} \\
\left.\begin{array}{ccc}
0 & 1 & 0 \\
-4 \pi^{2} & \frac{-\pi}{5} & 0 \\
0 & 0 & 0
\end{array}\right) \boldsymbol{u}_{k}-\left(\begin{array}{c}
0 \\
10 \sin (4 k h) \\
40 \cos (4 k h)
\end{array}\right)
\end{gathered}
$$

following $k$.

The results obtained using the $\Phi$-functions series method, are compared with the known codes:

LSODE methods, causes a numerical solution to be found using the Livermore Stiff Ode Solver. It solves stiff and nonstiff systems. It uses Adams methods (predictor-corrector) in the nonstiff case, and Backward Differentiation Formula (BDF) in the stiff case.

ROSENBROCK the method finds a numerical solution using an Implicit Rosenbrock third-fourth order Runge-Kutta method with degree three interpolant. 
GEAR causes a numerical solution to be found by way of Burlisch-Stöer rational extrapolation method. The method has higher precision and calculation efficiency, especially in solving stiff differential equations.

TAYLOR SERIES the method finds a numerical solution to the differential equations, using a Taylor series method. This method can be used for high accuracy solutions.

NEWMARK BETA-METHOD is a method of numerical integration used to solve differential equations. In this method the constant average acceleration is generally used in structural dynamics because it has been shown to have a high degree of numerical stability.

WILSON THETA-METHOD assumes that the acceleration of the system varies linearly between two instants of time, $t$ to $t+\theta h$, where the value of $\theta$ need not be an integer and is usually greater than 1.0. the method is unconditionally stable for linear dynamic systems when $\theta>1.37$, and a value of $\theta=1.4$ is often used for nonlinear dynamic systems.

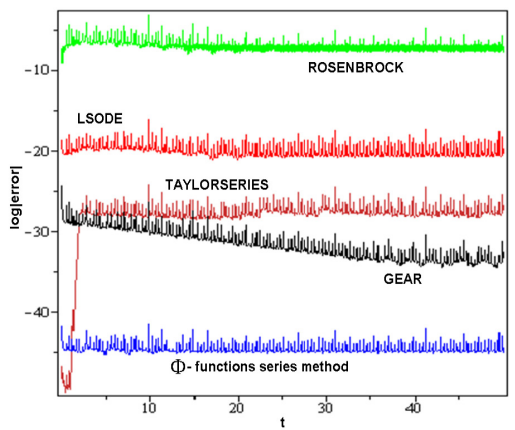

Figure 2: The decimal logarithm of module of the relative error of the solution $\boldsymbol{u}(t)$.

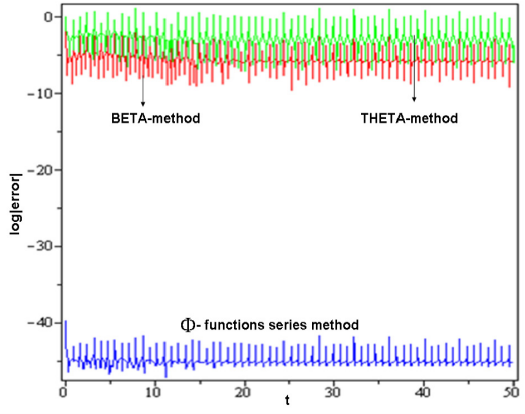

Figure 3: The decimal logarithm of the relative error of the solution $x(t)$.

Fig. 2 shows the graph of the decimal logarithm of module of the relative error of the solution $\boldsymbol{u}(t)$, vs $t$, calculate using (11), step size $h=0.01$ and 50 digits, with the numerical integration codes LSODE with tol $=10^{-25}$, ROSENBROCK with abserr $=10^{-30}$, GEAR with errorper $=10^{-25}$ and TAYLORSERIES with abserr $=10^{-25}$.

Fig. 3 shows the logarithm graph for the absolute value of the relative error of solution $x(t)$, vs. $t$, obtained with 50 digits, calculated by means of (11), with two $\Phi$-functions and step size $h=0.001$, compared with the numerical integration codes NEWMARK BETA-METHOD with $\delta=1 / 2, \alpha=1 / 4$, $h=0.001$ and WILSON THETA-METHOD with $\delta=1 / 2, \alpha=1 / 6, \theta=1.4$, $h=0.001$. Analogous results are obtained for the velocity $\dot{x}(t)$. 


\section{Application of the $\Phi$-functions series method to an earthquake modelled by a 2DOF}

A 2DOF can be represented in Fig. 4 and it is used to study the dynamic forces acting on this system. Similarly at SDOF, four types of forces act on each floor mass, the stiffness force, the damping force, the external force and inertial force [7].

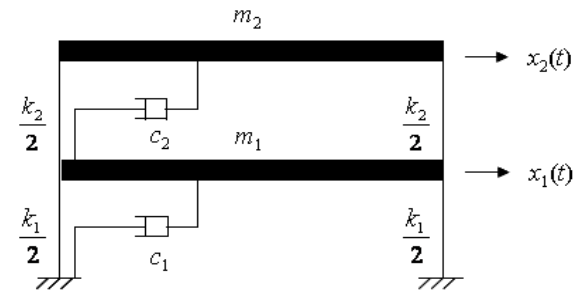

Figure 4: $\quad$ Two Degrees of Freedom System (2DOF).

The dynamic equilibrium equations of motion are:

$$
\left(\begin{array}{cc}
m_{1} & 0 \\
0 & m_{2}
\end{array}\right)\left(\begin{array}{l}
\ddot{x}_{1} \\
\ddot{x}_{2}
\end{array}\right)+\left(\begin{array}{cc}
c_{1}+c_{2} & -c_{2} \\
-c_{2} & c_{2}
\end{array}\right)\left(\begin{array}{l}
\dot{x}_{1} \\
\dot{x}_{2}
\end{array}\right)+\left(\begin{array}{cc}
k_{1}+k_{2} & -k_{2} \\
-k_{2} & k_{2}
\end{array}\right)\left(\begin{array}{l}
x_{1} \\
x_{2}
\end{array}\right)=\left(\begin{array}{l}
F_{1}(t) \\
F_{2}(t)
\end{array}\right)
$$

defining $\quad M=\left(\begin{array}{cc}m_{1} & 0 \\ 0 & m_{2}\end{array}\right), \quad C=\left(\begin{array}{cc}c_{1}+c_{2} & -c_{2} \\ -c_{2} & c_{2}\end{array}\right), \quad K=\left(\begin{array}{cc}k_{1}+k_{2} & -k_{2} \\ -k_{2} & k_{2}\end{array}\right), \boldsymbol{x}(t)=\left(\begin{array}{l}x_{1}(t) \\ x_{2}(t)\end{array}\right) \quad$ and $F(t)=\left(\begin{array}{ll}F_{1}(t) & F_{2}(t)\end{array}\right)^{t}$, when the symmetrical and positive definite matrices $M, C$ and $K$, are the mass, damping and stiffness matrix, respectively; the system (12) can be expressed by $M \ddot{\boldsymbol{x}}(t)+C \dot{\boldsymbol{x}}(t)+K \boldsymbol{x}(t)=F(t)$.

Considering that the structure is subjected to an earthquake ground motion, where only horizontal translation of the earthquake ground motion is considered. Applying the Newton's second law and given that the external force is zero, are:

$$
\begin{aligned}
& m_{1} \ddot{y}_{1}+c_{2}\left(\dot{y}_{1}-\dot{y}_{2}\right)+c_{1}\left(\dot{y}_{1}-\dot{u}_{g}\right)+k_{2}\left(y_{1}-y_{2}\right)+c_{1}\left(y_{1}-u_{g}\right)=0, \\
& m_{2} \ddot{y}_{2}+c_{2}\left(\dot{y}_{2}-\dot{y}_{1}\right)+k_{2}\left(y_{2}-y_{1}\right)=0,
\end{aligned}
$$

where $u_{g}$ and $\dot{u}_{g}$ are the absolute ground displacement and the absolute ground velocity, respectively.

If it define $x_{1}(t)=y_{1}(t)-u_{g}(t)$ and $x_{2}(t)=y_{2}(t)-u_{g}(t), \quad$ as relative displacement between the mass and the ground, the equations (13) are:

$$
\left(\begin{array}{cc}
m_{1} & 0 \\
0 & m_{2}
\end{array}\right)\left(\begin{array}{l}
\ddot{x}_{1} \\
\ddot{x}_{2}
\end{array}\right)+\left(\begin{array}{cc}
c_{1}+c_{2} & -c_{2} \\
-c_{2} & c_{2}
\end{array}\right)\left(\begin{array}{l}
\dot{x}_{1} \\
\dot{x}_{2}
\end{array}\right)+\left(\begin{array}{cc}
k_{1}+k_{2} & -k_{2} \\
-k_{2} & k_{2}
\end{array}\right)\left(\begin{array}{l}
x_{1} \\
x_{2}
\end{array}\right)=-\left(\begin{array}{cc}
m_{1} & 0 \\
0 & m_{2}
\end{array}\right)\left(\begin{array}{l}
1 \\
1
\end{array}\right) \ddot{u_{g}}
$$

If $\left(\begin{array}{cc}m_{1} & 0 \\ 0 & m_{2}\end{array}\right)\left(\begin{array}{l}1 \\ 1\end{array}\right) \ddot{u}_{g}$ is a harmonic matrix forcing function, i.e.:

$$
\left(\begin{array}{cc}
m_{1} & 0 \\
0 & m_{2}
\end{array}\right)\left(\begin{array}{l}
1 \\
1
\end{array}\right) \ddot{u}_{g}=\left(\begin{array}{l}
F_{0} \sin \left(\omega_{0} t\right) \\
F_{0} \sin \left(\omega_{0} t\right)
\end{array}\right)
$$


equation (14) is:

$$
\left(\begin{array}{cc}
m_{1} & 0 \\
0 & m_{2}
\end{array}\right)\left(\begin{array}{l}
\ddot{x}_{1} \\
\ddot{x}_{2}
\end{array}\right)+\left(\begin{array}{cc}
c_{1}+c_{2} & -c_{2} \\
-c_{2} & c_{2}
\end{array}\right)\left(\begin{array}{l}
\dot{x}_{1} \\
\dot{x}_{2}
\end{array}\right)+\left(\begin{array}{cc}
k_{1}+k_{2} & -k_{2} \\
-k_{2} & k_{2}
\end{array}\right)\left(\begin{array}{l}
x_{1} \\
x_{2}
\end{array}\right)=-\left(\begin{array}{l}
F_{0} \sin \left(\omega_{0} t\right) \\
F_{0} \sin \left(\omega_{0} t\right)
\end{array}\right)
$$

at the moment that the earthquake occurs, it is very reasonable to assume that the structure is at rest.

Therefore or normalized form, the IVP is:

$$
\begin{aligned}
& \ddot{\boldsymbol{x}}(t)+M^{-1} C \dot{\boldsymbol{x}}(t)+M^{-1} K \boldsymbol{x}(t)=-\left(F_{0} \sin \left(\omega_{0} t\right) / m_{1} \quad F_{0} \sin \left(\omega_{0} t\right) / m_{2}\right)^{t} \\
& \boldsymbol{x}(0)=\boldsymbol{x}_{0}=\left(\begin{array}{ll}
0 & 0
\end{array}\right)^{t} \text { and } \dot{\boldsymbol{x}}(0)=\dot{\boldsymbol{x}}_{0}=\left(\begin{array}{ll}
0 & 0
\end{array}\right)^{t}
\end{aligned}
$$

In order to apply the $\Phi$-function series method, is effected the change of variable: $x_{1}=u_{1}, \dot{x}_{1}=u_{3}, \ddot{x}_{1}=\dot{u}_{3}, x_{2}=u_{2}, \dot{x}_{2}=u_{4}, \ddot{x}_{2}=\dot{u}_{4}$.

The IVP (17) can be expressed as:

$$
\left(\begin{array}{l}
\dot{u}_{1} \\
\dot{u}_{2} \\
\dot{u}_{3} \\
\dot{u}_{4}
\end{array}\right)+\left(\begin{array}{cc}
O_{2 \times 2} & I_{2 \times 2} \\
M^{-1} K & M^{-1} C
\end{array}\right)\left(\begin{array}{l}
u_{1} \\
u_{2} \\
u_{3} \\
u_{4}
\end{array}\right)=-\left(\begin{array}{llll}
0 & 0 & \frac{F_{0} \sin \left(\omega_{0} t\right)}{m_{1}} & \frac{F_{0} \sin \left(\omega_{0} t\right)}{m_{2}}
\end{array}\right)^{t}, \boldsymbol{u}(0)=\left(\begin{array}{l}
0 \\
0 \\
0 \\
0
\end{array}\right) .
$$

\subsection{Resolution of a seismic model (2DOF) by $\Phi$-functions series method}

Consider the two-story frame subjected to an earthquake ground motion [7] (Fig. 5).

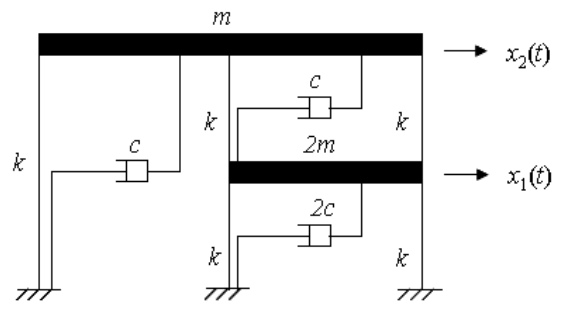

Figure 5: Two-story frame.

The dynamic equilibrium equation of motion is:

$$
\left(\begin{array}{cc}
2 m & 0 \\
0 & m
\end{array}\right)\left(\begin{array}{l}
\ddot{x}_{1} \\
\ddot{x}_{2}
\end{array}\right)+\left(\begin{array}{cc}
3 c & -c \\
-c & 2 c
\end{array}\right)\left(\begin{array}{c}
\dot{x}_{1} \\
\dot{x}_{2}
\end{array}\right)+\left(\begin{array}{cc}
4 k & -2 k \\
-2 k & 3 k
\end{array}\right)\left(\begin{array}{l}
x_{1} \\
x_{2}
\end{array}\right)=-\left(\begin{array}{cc}
2 m & 0 \\
0 & m
\end{array}\right)\left(\begin{array}{l}
1 \\
1
\end{array}\right) \ddot{u}_{g}(t)
$$

If $\left(\begin{array}{cc}2 m & 0 \\ 0 & m\end{array}\right)\left(\begin{array}{l}1 \\ 1\end{array}\right) \ddot{u}_{g}(t)$ is a harmonic matrix forcing function, i.e. $\left(\begin{array}{cc}2 m & 0 \\ 0 & m\end{array}\right)\left(\begin{array}{l}1 \\ 1\end{array}\right) \ddot{u}_{g}(t)=\left(\begin{array}{l}F_{0} \sin \left(\omega_{0} t\right) \\ F_{0} \sin \left(\omega_{0} t\right)\end{array}\right)$ then equation (19) is:

$$
\left(\begin{array}{cc}
2 m & 0 \\
0 & m
\end{array}\right)\left(\begin{array}{l}
\ddot{x}_{1} \\
\ddot{x}_{2}
\end{array}\right)+\left(\begin{array}{cc}
3 c & -c \\
-c & 2 c
\end{array}\right)\left(\begin{array}{l}
\dot{x}_{1} \\
\dot{x}_{2}
\end{array}\right)+\left(\begin{array}{cc}
4 k & -2 k \\
-2 k & 3 k
\end{array}\right)\left(\begin{array}{l}
x_{1} \\
x_{2}
\end{array}\right)=-\left(\begin{array}{l}
F_{0} \sin \left(\omega_{0} t\right) \\
F_{0} \sin \left(\omega_{0} t\right)
\end{array}\right)
$$

In notation more compact and normalizing the equation (20), is obtained: 


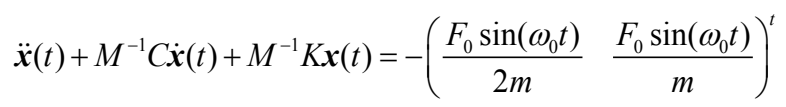

at the moment that the earthquake occurs, it is very reasonable to assume that the structure is at rest.

To solve the IVP:

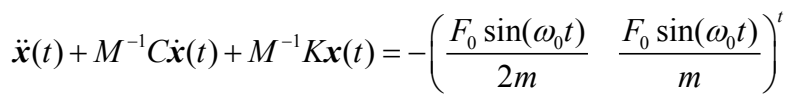

with $\boldsymbol{x}(0)=0, \dot{\boldsymbol{x}}(0)=0, t \in[0, T]$,

using the methodology of the $\Phi$-functions, the new expression for the IVP

$$
\left(\begin{array}{c}
\dot{u}_{1}(t) \\
\dot{u}_{2}(t) \\
\dot{u}_{3}(t) \\
\dot{u}_{4}(t)
\end{array}\right)+\left(\begin{array}{cc}
O_{2 \times 2} & I_{2 \times 2} \\
M^{-1} K & M^{-1} C
\end{array}\right)\left(\begin{array}{c}
u_{1}(t) \\
u_{2}(t) \\
u_{3}(t) \\
u_{4}(t)
\end{array}\right)=-\left(\begin{array}{llll}
0 & 0 & \frac{F_{0}}{2 m} \sin \left(\omega_{0} t\right) & \frac{F_{0}}{m} \sin \left(\omega_{0} t\right)
\end{array}\right)^{t},
$$

$\boldsymbol{u}(0)=\mathbf{0}$ where $M^{-1} K=\frac{k}{m}\left(\begin{array}{cc}2 & -1 \\ -2 & 3\end{array}\right)$ and $M^{-1} C=\frac{c}{2 m}\left(\begin{array}{cc}3 & -1 \\ -2 & 4\end{array}\right)$.

The variable is introduced in order to make easier the elimination the disturbance's function of the IVP (23), following the Steffensen's techniques $[8,9]$.

$$
\begin{aligned}
& u_{5}=-\frac{F_{0}}{2 m} \sin \left(\omega_{0} t\right) \text {, obtaining a new IVP. }
\end{aligned}
$$

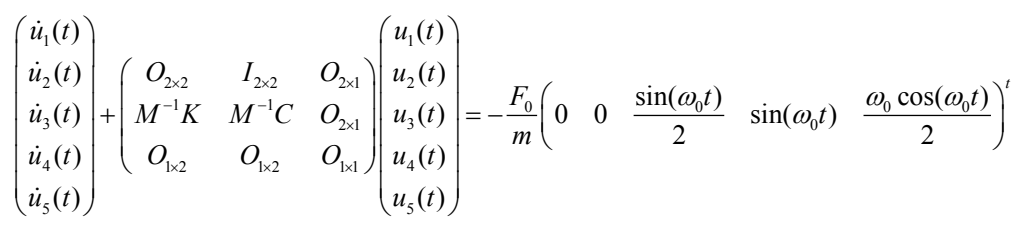

with $\boldsymbol{u}(0)=\mathbf{0}$.

To invalidate the function of disturbance, the differential operator $(D+B)$ is applied to (24), where $B$ is the following matrix

$$
B=\left(\begin{array}{ccc}
O_{2 \times 2} & O_{2 \times 2} & O_{2 \times 1} \\
O_{2 \times 2} & O_{2 \times 2} & \bar{\Omega}_{2 \times 1} \\
O_{1 \times 2} & \Omega_{1 \times 2} & O_{1 \times 1}
\end{array}\right) \text {, with } \bar{\Omega}_{2 \times 1}=\left(\begin{array}{c}
-1 \\
-2
\end{array}\right) \text { and } \Omega_{1 \times 2}=\left(\begin{array}{ll}
\omega_{0}^{2} & 0
\end{array}\right)
$$

obtaining the extended IVP

$$
\left(\begin{array}{l}
\ddot{u}_{1}(t) \\
\ddot{u}_{2}(t) \\
\ddot{u}_{3}(t) \\
\ddot{u}_{4}(t) \\
\ddot{u}_{5}(t)
\end{array}\right)+\left(\begin{array}{ccc}
O_{2 \times 2} & I_{2 \times 2} & O_{2 \times 1} \\
M^{-1} K & M^{-1} C & \bar{\Omega}_{2 \times 1} \\
O_{1 \times 2} & \Omega_{1 \times 2} & O_{1 \times 1}
\end{array}\right)\left(\begin{array}{l}
\dot{u}_{1}(t) \\
\dot{u}_{2}(t) \\
\dot{u}_{3}(t) \\
\dot{u}_{4}(t) \\
\dot{u}_{5}(t)
\end{array}\right)+\left(\begin{array}{ccc}
O_{2 \times 2} & O_{2 \times 2} & O_{2 \times 1} \\
O_{2 \times 2} & O_{2 \times 2} & O_{2 \times 1} \\
\Omega_{1 \times 2} M^{-1} K & \Omega_{1 \times 2} M^{-1} C & O_{1 \times 1} \\
u_{2}(t) \\
u_{3}(t) \\
u_{4}(t) \\
u_{5}(t)
\end{array}\right)=\left(\begin{array}{l}
u_{1}(t) \\
0 \\
0 \\
0 \\
0
\end{array}\right),
$$




$$
\boldsymbol{u}(0)=\left(\begin{array}{lllll}
0 & 0 & 0 & 0 & 0
\end{array}\right)^{t}, \dot{\boldsymbol{u}}(0)=\left(\begin{array}{lllll}
0 & 0 & 0 & 0 & -\frac{F_{0}}{2 m} \omega_{0}
\end{array}\right)^{t},
$$

which is integrated exactly using the $\Phi$-functions series algorithm described in [6].

Choosing the following values for the structural variables [7]:

$$
m=1.0 \frac{\mathrm{k} \cdot \mathrm{s}^{2}}{\mathrm{in} .}, \zeta=5 \%, \omega_{n}=2 \pi \frac{\mathrm{rad}}{\mathrm{s}}, F_{0}=10 \mathrm{kip}, \omega_{0}=4 \frac{\mathrm{rad}}{\mathrm{s}} \text { and } T=1 \mathrm{~s} .
$$

The IVP is:

$$
\left(\begin{array}{ll}
2 & 0 \\
0 & 1
\end{array}\right)\left(\begin{array}{l}
\ddot{x}_{1} \\
\ddot{x}_{2}
\end{array}\right)+\left(\begin{array}{rr}
\frac{3 \pi}{10} & -\frac{\pi}{5} \\
-\frac{\pi}{5} & \frac{2 \pi}{5}
\end{array}\right)\left(\begin{array}{l}
\dot{x}_{1} \\
\dot{x}_{2}
\end{array}\right)+\left(\begin{array}{cc}
16 \pi^{2} & -8 \pi^{2} \\
-8 \pi^{2} & 12 \pi^{2}
\end{array}\right)\left(\begin{array}{l}
x_{1} \\
x_{2}
\end{array}\right)=-\left(\begin{array}{l}
10 \sin (4 t) \\
10 \sin (4 t)
\end{array}\right),\left(\begin{array}{l}
x_{1}(0) \\
x_{2}(0)
\end{array}\right)=\left(\begin{array}{l}
0 \\
0
\end{array}\right)
$$

making the change of variable $x_{1}=u_{1}, \dot{x}_{1}=u_{3}, \ddot{x}_{1}=\dot{u}_{3}$ and $x_{2}=u_{2}, \dot{x}_{2}=u_{4}$, $\ddot{x}_{2}=\dot{u}_{4}$, the new IVP is:

$$
\left(\begin{array}{l}
\dot{u}_{1}(t) \\
\dot{u}_{2}(t) \\
\dot{u}_{3}(t) \\
\dot{u}_{4}(t) \\
\dot{u}_{5}(t)
\end{array}\right)+\left(\begin{array}{ccccc}
0 & 0 & -1 & 0 & 0 \\
0 & 0 & 0 & -1 & 0 \\
8 \pi^{2} & -4 \pi^{2} & \frac{3 \pi}{10} & \frac{-\pi}{10} & 0 \\
-8 \pi^{2} & 12 \pi^{2} & \frac{-\pi}{5} & \frac{2 \pi}{5} & 0 \\
0 & 0 & 0 & 0 & 0
\end{array}\right)\left(\begin{array}{l}
u_{1}(t) \\
u_{2}(t) \\
u_{3}(t) \\
u_{4}(t) \\
u_{5}(t)
\end{array}\right)=-\left(\begin{array}{c}
0 \\
0 \\
5 \sin (4 t) \\
10 \sin (4 t) \\
20 \cos (4 t)
\end{array}\right),\left(\begin{array}{l}
u_{1}(0) \\
u_{2}(0) \\
u_{3}(0) \\
u_{4}(0) \\
u_{5}(0)
\end{array}\right)=\left(\begin{array}{l}
0 \\
0 \\
0 \\
0 \\
0
\end{array}\right) .
$$

A matrix which annihilates the disturbance function is:

$$
B=\left(\begin{array}{ccccc}
0 & 0 & 0 & 0 & 0 \\
0 & 0 & 0 & 0 & 0 \\
0 & 0 & 0 & 0 & -1 \\
0 & 0 & 0 & 0 & -2 \\
0 & 0 & 16 & 0 & 0
\end{array}\right)
$$

applying the operator $(D+B)$ to the system (28) we obtain the extended IVP:

$$
\begin{gathered}
\left(\begin{array}{l}
\ddot{u}_{1}(t) \\
\ddot{u}_{2}(t) \\
\ddot{u}_{3}(t) \\
\ddot{u}_{4}(t) \\
\ddot{u}_{5}(t)
\end{array}\right)+\left(\begin{array}{ccccc}
0 & 0 & 1 & 0 & 0 \\
0 & 0 & 0 & 1 & 0 \\
8 \pi^{2} & -4 \pi^{2} & \frac{3 \pi}{10} & \frac{-\pi}{10} & -1 \\
-8 \pi^{2} & 12 \pi^{2} & \frac{-\pi}{5} & \frac{2 \pi}{5} & -2 \\
0 & 0 & 16 & 0 & 0
\end{array}\right)\left(\begin{array}{l}
\dot{u}_{1}(t) \\
\dot{u}_{2}(t) \\
\dot{u}_{3}(t) \\
\dot{u}_{4}(t) \\
\dot{u}_{5}(t)
\end{array}\right)+\left(\begin{array}{ccccc}
0 & 0 & 0 & 0 & 0 \\
0 & 0 & 0 & 0 & 0 \\
0 & 0 & 0 & 0 & 0 \\
0 & 0 & 0 & 0 & 0 \\
128 \pi^{2} & -64 \pi^{2} & \frac{24 \pi}{5} & \frac{-8 \pi}{5} & 0
\end{array}\right)\left(\begin{array}{l}
u_{1}(t) \\
u_{2}(t) \\
u_{3}(t) \\
u_{4}(t) \\
u_{5}(t)
\end{array}\right)=\left(\begin{array}{l}
0 \\
0 \\
0 \\
0 \\
0
\end{array}\right), \\
\boldsymbol{u}(0)=\left(\begin{array}{lllll}
0 & 0 & 0 & 0 & 0
\end{array}\right)^{t}, \dot{\boldsymbol{u}}(0)=\left(\begin{array}{lllll}
0 & 0 & 0 & 0 & -20
\end{array}\right)^{t},
\end{gathered}
$$

that is integrated exactly by the following algorithm the $\Phi$-functions series , applied to this problem

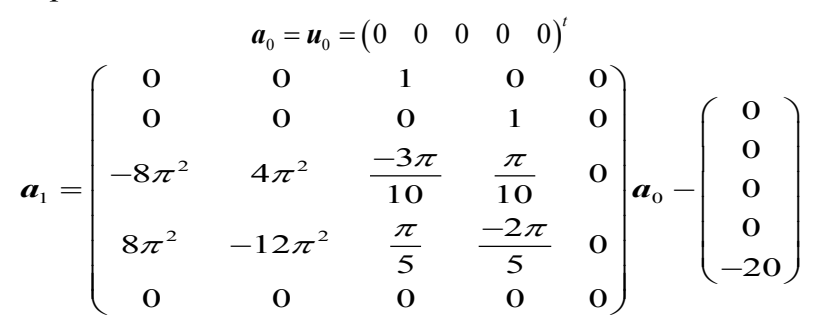


from $\mathrm{k}=1$ up to $\mathrm{n}$ calculates:

$$
\begin{gathered}
\boldsymbol{u}_{k}=\Phi_{0}(h) \boldsymbol{a}_{0}+\Phi_{1}(h) \boldsymbol{a}_{1} \\
\boldsymbol{a}_{1}=\left(\begin{array}{ccccc}
0 & 0 & 1 & 0 & 0 \\
0 & 0 & 0 & 1 & 0 \\
-8 \pi^{2} & 4 \pi^{2} & \frac{-3 \pi}{10} & \frac{\pi}{10} & 0 \\
8 \pi^{2} & -12 \pi^{2} & \frac{\pi}{5} & \frac{-2 \pi}{5} & 0 \\
0 & 0 & 0 & 0 & 0
\end{array}\right) \boldsymbol{u}_{k}-\left(\begin{array}{c}
0 \\
0 \\
5 \sin (4 k h) \\
10 \sin (4 k h) \\
20 \cos (4 k h)
\end{array}\right)
\end{gathered}
$$

following $k$.

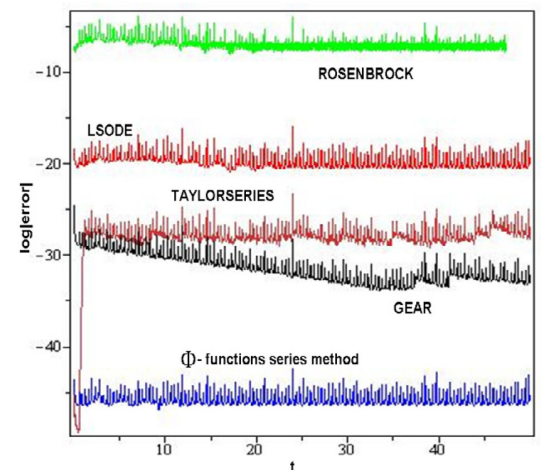

Figure 6: The decimal logarithm error of the solution $\boldsymbol{u}(t)$.

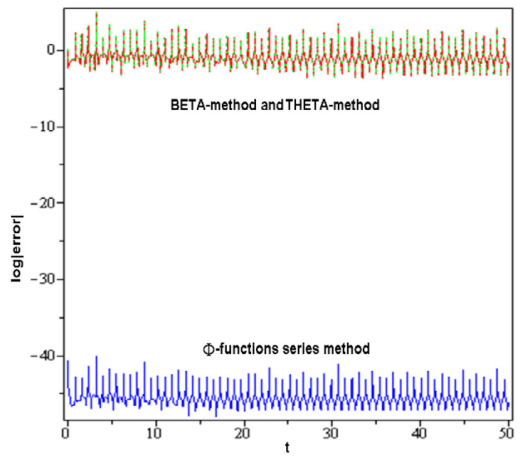

Figure 7: The decimal logarithm of module of the relative error of the position $\boldsymbol{x}(t)$.

Fig. 6 shows the graph of the decimal logarithm of module of the relative error of the solution $\boldsymbol{u}(t)$, vs $t$, calculate using (31), step size $h=0.01$ and 50 digits, with the numerical integration codes LSODE with tol $=10^{-25}$, ROSENBROCK with abserr $=10^{-30}$, GEAR with errorper $=10^{-25}$ and TAYLORSERIES with abserr $=10^{-25}$.

Fig. 7 shows the graph of the decimal logarithm of module of the relative error of the solution $\boldsymbol{x}(t)$, vs $t$, obtained with 50 digits, calculated by means of (31), with two $\Phi$-functions and step size $h=0.001$, compared with the numerical integration codes NEWMARK BETA-METHOD with $\delta=1 / 2$, $\alpha=1 / 4, h=0.001$ and WILSON THETA-METHOD with $\delta=1 / 2, \alpha=1 / 6$, $\theta=1.4, h=0.001$. Analogous results are obtained for the velocity $\dot{\boldsymbol{x}}(t)$. 


\section{Conclusions}

The method of numerical integration of $\Phi$-functions series is based on the ideas developed by Scheifele in his $\Gamma$-functions series method. The $\Phi$-functions series method has an advantage over the Scheifele method to integrate exactly the perturbed problem, transforming it into second-order homogeneous problem which is able to integrate exactly with two first $\Phi$-functions.

The good performance and accuracy of the $\Phi$-functions series method is shown by comparing numerical results obtained in the resolution of a SDOF and 2DOF with the results calculated with other well known integrators, such as ROSENBROCK, GEAR, TAYLORSERIES, NEWMARK BETA-METHOD and WILSON THETA-METHOD.

\section{Acknowledgement}

This work has been supported by the project of the Generalitat Valenciana $\mathrm{GV} / 2011 / 032$.

\section{References}

[1] E. L. Stiefel. and G. Scheifele, "Linear and Regular Celestial Mechanics", Springer, Berlin-Heldelberg-New York, 1971.

[2] G. Scheifele, "On numerical integration of perturbed linear oscillating systems”, ZAMP. Vol. 22, 1971, pp. 186-210.

[3] J. A. Reyes, F. García-Alonso, J.M. Ferrándiz and J. Vigo-Aguiar, "Numeric multistep variable methods for perturbed linear system integration", Appl. Math. Comput, Vol. 190, 2007, pp. 63-79.

[4] F. García-Alonso, J. A. Reyes, J.M. Ferrándiz and J. Vigo-Aguiar, "Accurate numerical integration of perturbed oscillatory Systems in two frequencies", Transactions on Mathematical Software TOMS, 36 (4), 2009, article 21.

[5] J.A. Reyes and F. García-Alonso, "Computational series and multistep methods to integrate forced and damped stiff oscillators", The Open Applied Mathematics Journal Vol. 6, 2012, pp. 9-22.

[6] F. García-Alonso and J.A. Reyes, "A new approach for exact integration of some perturbed stiff linear systems of oscillatory type", Appl. Math. Comput. Vol. 215, 2009, pp. 2649-2662.

[7] G. C. Hart and K. Wong, "Structural dynamics for structural engineers", John Wiley \& Sons, Inc., 1999.

[8] J. F. Steffensen, "On the differential equations of Hill in the theory of the motion of the moon". Acta Math. Vol. 93, 1955, pp.169-177.

[9] J. F. Steffensen, "On the differential equations of Hill in the theory of the motion of the moon II", Acta Math. Vol. 95, 1955, pp. 25-37. 